\title{
El tratamiento psicológico de la tricotilomanía: Un estudio de caso
}

\author{
Teresa Sarmiento ${ }^{1}$, Verónica Guillen ${ }^{2,3,4}$ y José $\mathrm{M}^{\mathrm{a}}$ Sánchez ${ }^{1}$ \\ ${ }^{1}$ Servicio de Salud Mental y Toxicomanías. Hospital de Mollet, Barcelona \\ ${ }^{2}$ Universidad Jaume I, Castellón, España \\ ${ }^{3}$ Centro Clínico PREVI, Castellón, España \\ ${ }^{4}$ Caber Fisiopatología Obesidad y Nutrición CB06/03 Instituto Carlos III, España
}

\begin{abstract}
Resumen: En este trabajo se presenta un caso clínico de tricotilomanía, cuyo objetivo es presentar en detalle el procedimiento seguido en un contexto de sanidad pública, utilizando técnicas cognitivo-comportamentales, para profundizar en el conocimiento de la eficacia de estos procedimientos de tratamiento y mostrar la viabilidad de su aplicación. Los resultados obtenidos muestran mejorías importantes en distintos aspectos clínicos: en primer lugar, desapareció completamente la conducta de arrancamiento del cabello, además disminuyó considerablemente la ansiedad y se normalizó el estado de ánimo. Todo ello nos permite llegar a la conclusión de que la intervención resultó satisfactoria.
\end{abstract}

Palabas clave: Tricotilomanía; tratamiento psicológico cognitivo conductual; diseminación tratamientos psicológicos; estudio de caso.

\section{Psychological treatment of trichotillomania: A case study}

\begin{abstract}
This paper presents a clinical case of trichotillomania, thus aims to present in detail the procedure followed in a public health context, using cognitive-behavioral techniques, in order to deepen the knowledge of the efficacy of these treatment procedures and demonstrate the feasibility of their use. The results obtained showed significant improvements in different clinical aspects: first, the hair pulling behavior disappeared completely; moreover, anxiety diminished significantly and mood normalized. This allows us to conclude that the intervention was successful.
\end{abstract}

Keywords: Trichotillomania; cognitive behavioral psychological treatment: psychological treatment dissemination; case study.

\section{Introducción}

Este problema fue descrito por primera vez por el médico francés Francois Henri Hallopeau (1989). Etimológicamente proviene del griego Trichos (pelo), tylos (estirar) y manía impulso. La tricotilomanía en el DSMIV estaba dentro de los Trastornos de control de impulsos, ahora se denomina Trastorno de tricotilomanía o de arrancarse el pelo, y se incluye en el DSM-5 dentro de los trastornos obsesivo - compulsivos y relacionados (American Psychiatric Association, 2014) que establece

Recibido: 30 agosto 2015; aceptado: 7 octubre 2015

Correspondencia: Teresa Sarmiento Luque. C/Paseo de la Habana, $\mathrm{N}^{\circ}$ 15-17, Esc. C-4, 6º $2^{\mathrm{a}}$. 08030. Barcelona, España. Correo-e: tsarmientoluque@gmail.com los siguientes criterios: arrancamiento del pelo de forma recurrente, lo que da lugar a su pérdida; este arrancamiento del pelo causa malestar clínicamente significativo o deterioro en lo social, laboral u otras áreas importantes del funcionamiento y existen intentos repetidos de disminuir o dejar de arrancarse el pelo.

La característica esencial de la tricotilomanía es el arrancamiento recurrente del cabello que da lugar a una pérdida perceptible de pelo. Puede haber arrancamiento del pelo en cualquier región del cuerpo donde este crezca (incluyendo las regiones axilar, púbica y perirrectal), pero los sitios más frecuentes son la cabeza, existiendo predilección por las regiones coronales o parietales, en el 80-90\% de los casos, (Hallopeu, 1989; Papadopoulos, Janniger, y Schwartz, 2003), las cejas y las pestañas (pueden llegar a desaparecer por completo). Una perso- 
na puede arrancarse el pelo de un único lugar; pero también puede hacerlo de distintos sitios. El arrancamiento del pelo no se produce en presencia de otras personas, regularmente ocultan su conducta frente a sus familiares más cercanos, evitando así la culpabilidad, degradación y rechazo (Pérez-Elizondo, Pineda-Pineda, y Arellano-Flores, 2012) y puede haber una evitación de situaciones sociales. Estas personas niegan frecuentemente su comportamiento y ocultan o camuflan la alopecia resultante (p. ej., mediante el uso de maquillaje o pelucas). Con el tiempo los afectados se suelen ir arrancando pelo de más sitios. Típicamente el pelo se arranca de uno en uno, aunque también se pueden arrancar en manojos (Duke, Keely, Geffken, y Storch, 2010). El arrancamiento de pelo puede ocurrir en episodios breves distribuidos a través del día o en menor frecuencia, pero de forma sostenida, continuar durante horas. Las circunstancias provocadoras de estrés aumentan el comportamiento de arrancamiento del pelo, aunque en los estados de relajación y distracción también se observa este comportamiento (p. ej., cuando se lee un libro o se ve la televisión). El acto de arrancarse el pelo también puede ir precedido o acompañado de diferentes estados emocionales. De este modo, puede provocarse por sentimientos de ansiedad o aburrimiento, puede estar precedido de una sensación de tensión creciente (ya sea inmediatamente antes de sacar el pelo o cuando se intenta resistir el impulso de arrancárselo). En algunos sujetos la tensión no precede necesariamente al acto, los dedos simplemente encuentran su camino hacia el sitio y comienzan el comportamiento. Muchas personas dicen que empiezan buscando alguna irregularidad concreta del cabello (por ejemplo que esté más grueso, con grumos), o en la piel (que esté más áspera, irregular, llena de imperfecciones). El acto de arrancarse el pelo puede implicar diversos grados de conciencia; algunas personas muestran una mayor atención al arrancarse el cabello (con una tensión previa y un alivio posterior), y otros individuos exhiben un comportamiento más automático (se tiran del pelo sin darse cuenta del todo). Muchos individuos refieren una mezcla de los dos estilos de comportamiento. Hay gratificación, bienestar o sensación de liberación cuando se ha arrancado el cabello. Algunas experimentan una sensación de "hormigueo" en la cabeza que es aliviada al arrancar el cabello. El dolor no suele acompañar a la extracción del cabello.

En la población general, la prevalencia anual estimada para la tricotilomanía en los adultos y los adolescentes está entre el 1y $2 \%$. Las mujeres se ven afectadas con mayor frecuencia que los varones, en un proporción de aproximadamente 10:1 (American Psychiatric Association, 2014). Lo que sí parecen indicar los estudios sobre el tema es que las mujeres lo sufren en mayor proporción que los hombres (Icart, Pulpon, y Icart, 2006; Moreno et al., 2007), aunque no todos los trabajos obtienen los mismos resultados (Duke, Bodzin, Tavares, Geffken, y Storch, 2009). Esto también puede reflejar diferencias en la búsqueda de tratamiento según el género o actitudes culturales en cuanto a la apariencia (p. ej., la aceptación de la pérdida de cabello es normal entre los varones). Cohen, Tein, y Simeon, (1995) señalan que un 40\% de los casos nunca se diagnostican y alrededor del $58 \%$ no recibe terapia especifica. Entre los niños con tricotilomanía, ambos sexos están representados por igual.

La tricotilomanía puede aparecer asociada a otras conductas repetitivas centradas en el cuerpo, como pellizcarse la piel, rascarse (costras, acné u otras imperfecciones, hacerse excoriaciones...) o morderse las uñas, los labios. El examen del pelo, el retorcimiento o la sujeción del pelo entre los dientes, y la tricofagia (comer cabellos). Algunas personas pueden arrancar el pelo de animales domésticos, muñecas y otros materiales fibrosos (p. ej., suéteres, alfombras). Además del elemento estético se pueden dar complicaciones médicas graves como la formación en diversas partes del intestino de bolas de pelo, que no pueden ser digeridas, pudiendo producirse obstrucción abdominal e incluso perforación. Las personas con tricotilomanía también pueden tener trastornos del estado de ánimo, trastornos de ansiedad o retraso mental. Cuando se sospecha de tricotilomanía y la persona niega los síntomas, los hallazgos de laboratorio, nos indican datos que pueden ayudar a realizar el diagnostico. La biopsia de las áreas afectadas puede revelar la presencia de pelos cortos y rotos. El examen histológico mostrará la presencia de folículos normales y lesionados en la misma área, así como un aumento del número de cabellos en crecimiento. Algunos folículos pilosos pueden presentar síntomas de traumatismo. La ausencia de inflamación distingue la alopecia provocada por tricotilomanía de la alopecia areata (es una enfermedad que afecta al folículo piloso y ocasiona parches redondos de pérdida de cabello, pudiendo llevar a la calvicie total).

En cuanto al origen del problema, es posible sostener que manipular el pelo se da también en los animales para acicalarse y es un signo de intimidad o de poder. En los humanos las raíces también podrían estar asociadas al acicalamiento, ya que el pelo constituye un atributo de belleza, sexualidad y fortaleza. Se convertiría en problema cuando la conducta se realiza de forma automática y fuera de control. Sin embargo, tirarse del pelo puede darse sin que se note la pérdida del pelo y sin que se asocie ningún malestar, esto indica que cabría considerarlo como un continuo que iría desde un hábito sin tras- 
cendencia ni consecuencias, hasta una tricotilomanía, que supone un problema psicológico importante, cuando desfigura a la persona y va asociado a un estrés notable (Duke, Keely, Storch., 2010).

La etiología de este problema es multidimensional y compleja (Kress, Kelly, y McCormick, 2004). Algunos clínicos (Castle, y Philips, 2006; Lochner et al., 2005) lo consideran un trastorno obsesivo compulsivo (el DSM-5 lo ha incluido en esta categoría diagnóstica), dado que los individuos refieren sentir un impulso incontrolable en muchos casos con una posterior sensación de decremento de tensión. Pero también se encuentra ausencia de pensamientos obsesivos en individuos que manifiestan de esta conducta en situaciones caracterizadas por el sedentarismo y la observación de la televisión, lectura..., se arrancan los pelos sin darse cuenta del todo, en vez de reducir la ansiedad o responder a una obsesión... Oranje y Peerebom (1986) sostienen que el estrés psicosocial dentro del marco familiar (mudanza a un nuevo hogar, reciente llegada de un nuevo hermano, hospitalización de la madre o debilidad en el desarrollo psicomotor) puede disparar el comienzo de la tricotilomanía. El estrés es considerado un factor precipitante (Reeve, Bernstein, y Christenson, 1992). Desde un punto de vista conductual (Azrin y Nunn, 1973; Kress et al., 2004) sugieren que la tricotilomanía es una respuesta al estrés, y como tal, los mecanismos conductuales que se dan son un condicionamiento clásico en el que determinados estímulos internos y externos, disparan la actividad de tirarse del pelo, como la posición del cuerpo o de la mano, o estar tumbado o arreglarse el pelo, mirarse al espejo, usar pinzas, estar solo, estar aburrido y se mantiene el hábito por reforzamiento operante, que surge de la reducción de tensión que produce el arrancamiento del pelo.

Según Swedo y Rapoport (1991), se diferencian dos formas clínico-evolutivas del padecimiento: la de inicio precoz, de los 5-8 años de vida, de curso episódico, benigno y autolimitado con períodos cortos de agudización y remisión. La otra, de comienzo tardío, crónica y discapacitante, afecta a los adolescentes y adultos jóvenes o de mediana edad, predomina en las mujeres. Algunas personas tienen síntomas de forma continua durante décadas, y en otros éstos pueden aparecer y desaparecer durante semanas, meses o años. Las áreas afectadas por el arrancamiento pueden variar a lo largo del tiempo. Otra clasificación es la realizada por Christenson, Mackenzie, y Mitchell (1991) y Christenson et al. (1993), que distinguen dos tipos: automático y consciente. Automático: se da sin conciencia de lo que se está haciendo, mientras se está concentrado en otras actividades, como pensar, estudiar, viendo TV etc. Afecta a tres de cada cuatro personas con tricotilomanía. Consciente: la persona se concentra en lo que está haciendo y no lo puede evitar. Se da en una de cada cuatro personas afectadas. Lo más frecuente es que los tipos consciente y automático coexistan. A nivel de tratamiento, hay estudios que indican que para el tipo automático el tratamiento más eficaz es la reversión de hábito (Azrin y Nunn, 1973), pero para el consciente es necesario añadir otras técnicas cognitivo-conductuales (Foa, Rothbaum, Riggs, y Murdock, 1991; Rothbaum, 1990).

En cuanto al diagnóstico diferencial deben considerarse otras causas de alopecia en las personas que niegan arrancarse el pelo (p. ej., alopecia areata, calvicie característica del hombre, lupus eritematoso discoide crónico, liquen plano folicular, foliculitis depilatorias, seudopelada y alopecia mucinosa). Por otra parte, no se debe realizar el diagnóstico de tricotilomanía si el comportamiento se explica mejor por la presencia de otro trastorno mental (p. ej., en respuesta a una idea delirante o a una alucinación en la esquizofrenia). El arrancamiento de pelo repetitivo en la tricotilomanía debe distinguirse de la compulsión, característica del trastorno obsesivo-compulsivo. En el trastorno obsesivo-compulsivo los comportamientos repetitivos son efectuados en respuesta a una obsesión, es decir, pueden arrancarse los pelos como parte de sus rituales de simetría. Tampoco se establecerá un diagnóstico adicional de trastorno de movimientos estereotipados si el comportamiento repetitivo se limita al arrancamiento del pelo. La alopecia provocada en la tricotilomanía debe distinguirse también del trastorno facticio con predominio de signos y síntomas físicos, en el que hay un propósito de asumir el papel de enfermo. Muchas personas retuercen y juegan con el pelo, especialmente durante los estados de elevada ansiedad, pero este comportamiento no basta para establecer el diagnóstico de tricotilomanía. En los niños son frecuentes los períodos cortos de arrancamiento del pelo, y éstos deben ser considerados un "hábito" temporal. Esta forma de arrancamiento del pelo en la infancia difiere de las formas de tricotilomanía en adultos, en que puede haber una ausencia de la tensión o el alivio asociado al arrancamiento. Por esto, entre los niños, debe reservarse el diagnóstico para casos en los que el comportamiento haya persistido durante varios meses.

Con respecto a la intervenciones terapéuticas utilizadas, las terapias cognitivas conductuales, han dado muestras de su efectividad (Duke, D.C., Keeley, M.L. Geffken, G.R. y Storch, E.A., 2010; Kraemer, 1999; Ninan, Rothbaum, Marsteller, Knight, y Eccard, 2000; Papadopouloset al., 2003, Pérez, 2004; van Minnen, Hoogduin, Keijsers, Hellenbrand, y Hendriks, 2003; Woods et al., 2006). El entrenamiento en relajación muscular progresiva de Jacobson, la toma de conciencia del compor- 
tamiento, la realización de conductas incompatibles, la inversión del hábito, la reestructuración cognitiva, las autoinstrucciones, el autorrefuerzo, y la exposición en imaginación e in vivo, han demostrado ser útiles en el tratamiento a través de diversos estudios (Himle, Flessner, y Woods, 2004; Enos y Plante, 2001; Diefenbach, Reitman, y Williamson, 2000; Elliott y Fuqua, 2000; Lerner, Franklin, Meadows, Hembree y Foa, 1998). En el marco de los tratamientos conductuales, la inversión de hábito propuesta por Azrin y Nunn (1973) ha demostrado su eficacia. La idea general de la reversión del hábito implica el aumento de darse cuenta por parte del paciente sobre cada ocurrencia del hábito y su interrupción por medio de una respuesta que compita con él, de forma que cuando se sea consciente de lo que se está haciendo se haga esa conducta para evitar tirarse del pelo (p.ej., apretar el puño).

Sin embargo, a pesar de estos avances en el tratamiento, sigue siendo un importante problema el ocultamiento que hacen los pacientes de la conducta, evitando en muchas ocasiones incluso el tratamiento, por vergüenza (Diefenbach, Tolin, Hannan, Crocetto y Worhunsky, 2005; Shusterman, Feld, Baer y Keuthen, 2009), o por la creencia de que no se puede hacer nada para tratar el problema (Shusterman et al., 2009; Walsh y McDougle, 2001). También existen diferencias importantes en las estrategias de tratamiento que se utilizan (Duke et al., 2010). Además teniendo en cuenta que un $40 \%$ de los casos nunca se diagnostican y alrededor del $58 \%$ no recibe terapia específica (Cohen et al., 1995), hace que nos plateemos presentar en detalle el procedimiento seguido en un caso de tricotilomanía, en un contexto de sanidad pública, utilizando técnicas cognitivo-comportamentales.

\section{Método}

\section{Participante}

Se trata de una mujer de 33 años de edad que acude a consulta por iniciativa propia, por el malestar suscitado en el contexto de diversos acontecimientos vitales estresantes en el contexto de diversos acontecimientos vitales estresantes en el último año y medio, relacionados con distintos ámbitos: la vivienda, problemas laborales y económicos. Está casada y vive con su marido. Estudió administración de empresa. Ha trabajado un tiempo de administrativa (refiere que llevaba tres meses trabajando y tras 15 días de baja laboral le despidieron). La paciente indica también que mantiene buenas relaciones familiares, sociales y con su pareja, que es su principal figura de apoyo.
Antecedentes personales somáticos: niega antecedentes de interés. Niega también consumo de tóxicos y alergias tóxico-medicamentosas conocidas. En cuanto a antecedentes familiares psiquiátricos, señala que su madre padeció depresión (fallecida hace 7 años). Respecto a antecedentes personales psiquiátricos, sobre $\operatorname{los} 7$ u 8 años estuvo un año sufriendo problemas de tricotilomanía (presentó alopecia) que cedieron sin tratamiento. Rasgos de personalidad pertenecientes al cluster B (agrupa un conjunto de síndromes que se caracterizan por unos patrones de conducta impulsivos, excesivamente dramáticos, emotivos e inestables).

Historia del problema: presenta empeoramiento vespertino, tendencia al aislamiento, disminución de la interacción social, mantiene actividades rutinarias y ve películas en casa para evadirse de todo, sensación de miedo inespecífico, insomnio de mantenimiento y, además, arrancamiento del pelo de la cabeza. Tiende a no revelarlo, al retirarse el pañuelo de la cabeza se evidencia alopecia en la zona temporo-parietal, verbaliza que cuando se arranca el pelo se tranquiliza. El problema se manifiesta con mayor virulencia en el contexto de diversos acontecimientos estresantes relacionados con la vivien$\mathrm{da}$, el ámbito laboral y el económico. Su casero tiene problemas con la herencia del mismo y los ha denunciado como "ocupas". En dicho contexto, se quedaron en el paro tanto su marido como ella, y han estado viviendo tres meses en casa de un familiar.

La aparición de la conducta de tricotilomanía se produjo hace cinco meses en el contexto de acontecimientos vitales estresantes. Manifiesta que antes de arrancarse el pelo siente tensión, picor y necesidad de arrancárselo, una vez lo hace se siente aliviada pero culpable. Describe episodios de aproximadamente unos 150 pelos, durante el intervalo de una o dos hora, cuando está sola en casa, "busco los gruesos, rugosos, los estiro, arranco, hago una bola y los tiro". Niega morderlos o comérselos. Respecto a la repercusión social lleva un pañuelo en la cabeza, evita salir de noche, lugares donde pueda haber gente que visualice su problema (por ejemplo, en el autobús se coloca en la parte más alta).

La paciente presenta rasgos de personalidad pertenecientes al Cluster B y también onicofagia (hábito compulsivo de comerse las uñas).

\section{Evaluación}

Para la recogida de datos se utilizaron los siguientes instrumentos:

Entrevista clínica. La evaluación de la tricotilomanía es dificultosa debido a que los individuos que la padecen 
tienen cierta tendencia a no querer revelarlo (Stein, Christenson y Hollander, 1999). La recogida de datos es fundamental si se quiere hacer un diagnóstico y tratamiento adecuados. La entrevista es una herramienta imprescindible a la hora de obtener información necesaria sobre distintos aspectos de este trastorno (Mulinari-Brenner y Bergfeld, 2001; Simeon y Favazza, 2001; Stein et al., 1999). Se formulan preguntas sobre la edad de inicio de la conducta problema, frecuencia del comportamiento, cantidad de vello arrancado, estado emocional (antes, durante y después de arrancarse el vello), estrategias empleadas para reducir o detener el comportamiento, soporte familiar, etc. También se exploran la respuesta física y la influencia de factores afectivos y cognitivos en dicho comportamiento (Stemberger, Stein, y Mansueto, 2003).

ISRA. Inventario de Situaciones y Repuestas de Ansiedad (Miguel-Tobal y Cano-Vindel, 2002). Evalúa la frecuencia de respuestas de ansiedad en el triple sistema de respuesta (cognitivas, fisiológicas y motoras) y en diferentes áreas situacionales (ansiedad de evaluación, interpersonal, fóbica y ante situaciones de la vida cotidiana). Está basado en el modelo interactivo multidimensional de la ansiedad de Endler y en el modelo tridimensional de Lang. La edad de aplicación es de 16 años en adelantes y la duración de la prueba es de aproximadamente cincuenta minutos. Tiene un coeficiente alfa de Cronbach de 0.92 .

MCMI-III. Inventario Clínico Multiaxial de Millon-III (Millon, 1994). Adaptación española por Cardenal, Sánchez y Ortiz-Tallo (2007). Proporciona información al profesional en tareas de evaluación y toma de decisiones de tratamiento sobre personas con dificultades emocionales e interpersonales. Evaluación de 4 escalas de control, 11 escalas básicas, 3 rasgos patológicos, 7 síndromes de gravedad moderada y 3 síndromes de gravedad severa. La edad de aplicación es a partir de 18 años y la duración de la misma oscila entre 20 y 30 minutos. Los coeficientes alfa de Cronbach en la muestra de tipificación española oscilan desde 0.65 en la escala 7 (Compulsivo) hasta 0.88 en la escala CC (Depresión mayor).

Autorregistro. El autorregistro (Tabla 1) es una característica clave de todos los programas de autocontrol (Herbert y Nelson, 1997). Este caso se utiliza con la finalidad de realizar un análisis funcional de la conducta y conocer la frecuencia de ocurrencia. El objetivo es identificar las relaciones entre la conducta, los antecedentes y las consecuencias como requisito previo al tratamiento (Stemberger et al., 2003).

\section{Objetivo}

El objetivo que se plantea es: reducir la frecuencia de arrancamiento del pelo.

\section{Hipótesis}

Se parte de la hipótesis de que la intervención cognitivo-conductual resultará eficaz para el tratamiento de la tricotilomanía.

\section{Intervención}

Se utilizó el procedimiento de la inversión de hábito propuesto por Azrin y Nunn, (1973). Dicho procedimiento tiene un número variable de componentes. Los tres componentes que se consideran más importantes son la toma de la conciencia, la respuesta de competencia y el apoyo social. La sensibilización o toma de conciencia consiste en ayudar a la persona a concentrarse en las circunstancias en las que es más probable que se arranque el pelo. Esto permite a las personas ser más conscientes de la probabilidad de que el comportamiento se produzca, y ofrece oportunidades para el empleo de técnicas terapéuticas destinadas a desalentar la realización de estas conductas problemáticas. La formación en respuesta de competencia se centra en enseñar al paciente a sustituir el arrancarse el cabello por otra respuesta que sea incompatible con el comportamiento no deseado. Por ejemplo, cuando el paciente experimenta el impulso de arrancarse el cabello en ese momento, cierra sus manos en un puño, resuelve el cubo de Rubik...Esta respuesta se repite cada vez que llega una situación donde hay deseo de arrancar el cabello.

Puesto que es posible que el entorno social no haya aconsejado adecuadamente, es conveniente realizar apoyo social que consiste en llevar a sus seres queridos y familiares al proceso de terapia con el fin de proporcionar durante la fase psicoeducativa del tratamiento la información necesaria para eliminar creencias irracionales ("Si dejo de hacerlo me dañaré de otra forma") y fomentar la retroalimentación positiva de los familiares cuando el individuo se involucre en respuestas de competencia. También se les puede enseñar el empleo de estrategias para dar aliento y recordatorios cuando el individuo está en una circunstancia en la que es probable que se arranque el pelo.

El tratamiento se basa en los siguientes módulos o contenidos:

(1) Se comienza con un análisis funcional de la conducta problema, determinando la conciencia con la que se realiza, las situaciones que la dis- 
paran, las cogniciones que la acompañan, las emociones que se evitan, etc. De acuerdo con ese resultado, se fijan los objetivos de la terapia.

(2) Motivación. Hay que tener en cuenta los aspectos que mantienen la conducta de arrancamiento del cabello y direccionar voluntariamente los comportamientos hacia la realización del objetivo.

(3) Conciencia. El hábito es en gran parte automático y, por tanto, ha de hacerse consciente para poder actuar y revertirlo. Los auto-registros son fundamentales en este paso, tanto para ser conscientes de cuando se están tirando del pelo, como de los pensamientos, sentimientos, sensaciones y emociones que acompañan a esta conducta.

(4) Respuesta de competencia: conducta incompatible con el hábito de arrancarse el pelo, aplicada preventiva y correctivamente. La conducta incompatible suele ser tensar los puños, lo que impide que se utilicen las manos para tirarse del pelo. Esta conducta incompatible se complementa con la aceptación de las emociones y sensaciones que acompañan al impulso de tirarse del pelo.

(5) Detectar las situaciones y comportamientos a los que se ha condicionado la tricotilomanía. Una vez identificados, el paciente se expone a ellos para entrenarse en dar la respuesta de competencia.

(6) Relajación y aceptación de las emociones. Se utilizan para manejar de otra forma las reacciones emocionales que se dan en este hábito.

(7) Práctica en la realidad y en la imaginación. Para ello se comienza provocando en sesión las situaciones similares a las que disparan la tricotilomanía, si fuera posible, y entrenando al paciente en todo el proceso que se ha planteado hasta ahora.

(8) Recuperación de actividades. La imagen que se tenía con la falta de pelo ha podido ir retirando a la persona de actividades que antes le eran reforzantes y por tanto es necesario volverlas a hacer.

(9) Registrar los progresos es un importante paso para fortalecer la motivación.

(10) Se trabaja en prevención de las recaídas.

Se realizaron ocho sesiones de tratamiento, con una frecuencia entre tres semanas y un mes y con una duración de cincuenta minutos.

Primera sesión: En la primera visita, se llevó a cabo la entrevista con el objetivo de recoger los datos relacio- nados con la anamnesis, datos familiares relevantes y relacionados con la historia problema y la conducta de arrancarse el pelo. El terapeuta y el paciente realizaron una evaluación exhaustiva de los comportamientos problemáticos, incluyendo pensamientos, sentimientos y conductas que se producían antes de escoger y arrancar, así mismo se realizó el autorregistro de los pensamientos, sentimientos y conductas mientras se arrancaba y, finalmente, aquello que la paciente realizaba después de realizar la conducta. Las técnicas que se utilizan en la intervención se muestran en la Tabla 2.

Segunda sesión: En una segunda visita se realizó una sesión psicoeducativa. Se administró también el ISRA. Se le pidió a la paciente registrar su comportamiento con el objetivo de establecer la línea base y, a partir de ahí, iniciar el entrenamiento en fomentar conciencia del hábito y reestablecer actividades distractoras (sacar el perro, salir con alguna amiga).

Tercera sesión: En la tercera sesión, la paciente relató cómo le había ido desde la última vista y se analizó el autorregistro conjuntamente con la terapeuta. Se había arrancado 163 pelos. A partir del registro, se comprueba que los episodios ocurren, sobre todo, cuando la paciente siente ansiedad, aburrimiento, en el contexto de estados emocionales negativos y sin la presencia de gente alrededor. La paciente puntualiza que las situaciones suelen ser cuando ve la TV, leyendo, hablando por teléfono...le precede una sensación de tensión y las consecuencias son sentimientos de alivio y después culpa, vergüenza (Tabla 1). En esa misma sesión, se llevó a cabo un entrenamiento en técnicas de respiración y relajación y se pautan como tareas para casa. Se continúa con la auto-observación y autorregistros, tratando de identificar de manera más específica las situaciones y lugares donde aumentaba o disminuía la conducta problema.

Cuarta sesión: En la cuarta sesión, al igual que en las anteriores se comenzó con el análisis de las dificultades encontradas en las tareas para casa y en la revisión del autorregistro conjuntamente para ayudar a la paciente a tomar conciencia de cuándo y cómo ocurre el problema. El registro pone de manifiesto que se ha producido un solo episodio de arrancamiento de 150 pelos (Tabla 1).

Posteriormente, en la sesión se sigue con el entrenamiento en respuesta alternativa, la reacción de competencia, se acuerda como reacción de competencia que cuando sienta el impulso agarrará el "cubo" y se pondrá a realizarlo, "tocará al perro", "tejerá usando los brazos como agujas", "escribirá". Como pautas para casa se establecen: continuar con la realización del autorregistro, practicar entrenamiento en relajación muscular de Jacobson, respiración diafragmática y la reacción de competencia. 
Tabla 1. Auto-registro

\begin{tabular}{|c|c|c|c|c|c|}
\hline Fecha & Situación & Antecedentes & $\begin{array}{l}\text { Respuesta (cognitiva, } \\
\text { fisiológica, conductual) }\end{array}$ & Consecuencias & $\mathrm{N}^{\mathrm{o}}$ pelos \\
\hline & $\begin{array}{l}\text { Sola: Estudiando, Viendo } \\
\text { la TV, } \\
\text { Leyendo, hablando por te- } \\
\text { léfono, le da su marido una } \\
\text { mala noticia.... }\end{array}$ & $\begin{array}{l}\text { Sensación de tensión, } \\
\text { hormigueo, picor. } \\
\text { Aburrimiento. } \\
\text { Preocupada, ansiosa }\end{array}$ & $\begin{array}{l}\text { "Me voy a un sitio donde } \\
\text { este sola". } \\
\text { "Dirijo la mano a la cabeza } \\
\text { y busco los gruesos, rugo- } \\
\text { sos, los estiro, arranco, } \\
\text { hago una bola y los tiro". } \\
\text { "Tirar del pelo". } \\
\text { "Ligera molestia tras el } \\
\text { arrancamiento" }\end{array}$ & $\begin{array}{l}\text { "Sentimientos culpa, ver- } \\
\text { güenza". } \\
\text { "Siento alivio". } \\
\text { "Tengo cada vez más zona } \\
\text { sin pelo". } \\
\text { "la gente me mira". } \\
\text { "Me tapo la cabezo con un } \\
\text { pañuelo". }\end{array}$ & $\begin{array}{l}163 \\
150\end{array}$ \\
\hline
\end{tabular}

Tabla 2. Técnicas cognitivo-conductuales usadas en la intervención

\begin{tabular}{ll}
\hline \multicolumn{1}{c}{ Objetivo } & \multicolumn{1}{c}{ Técnica } \\
\hline - Disminuir y controlar el nivel de ac- & - Entrenamiento en técnicas de respiración. \\
tivación fisiológica & - Entrenamiento en relajación muscular progresiva de Jacobson. \\
- Disminuir la intensidad y frecuencia & - Reestructuración cognitiva. \\
de los pensamientos negativos. & - Detención del pensamiento. \\
- Modificar las distorsiones cognitivas. & - Autoinstrucciones. \\
& - Autorrefuerzo. \\
- Aumentar la motivación para el & - Tomar conciencia de cuándo y cómo ocurre. \\
abandono del hábito. & - Exposiciones en imaginación y en in vivo de las mejorías ante la ausencia de la conducta \\
& problema. \\
- Disminuir la frecuencia de arranca- & - Conducta incompatible. Respuesta alternativa, reacción de competencia. \\
miento. & \\
Disminuir la frecuencia de las con- & - Explicación de la actuación del reforzamiento positivo y negativo en su conducta. \\
ductas de evitación. & - Resolución de problemas. \\
& - Autorrefuerzo. \\
& - Refuerzo del terapeuta. \\
\hline
\end{tabular}

Quinta sesión: En la quinta sesión se comenzó igual que las anteriores. En esta ocasión se comprueba que ha habido un episodio de arrancamiento de 46 pelos. Se le presenta a la paciente la técnica de exposición en imaginación, se identifican los pensamientos negativos para poner en práctica la detención del pensamiento y las auto-instrucciones. Se llevó a cabo un entrenamiento en auto-instrucciones positivas ("voy a dejar de tirarme del pelo", "este picor de cabeza no es normal, es lo que siento antes de empezar a arrancarme el pelo, continuo con lo que estoy haciendo", "tengo herramientas para manejar el este problema”, "esto se pasa", "puedo con ello"); y un entrenamiento en estrategias de detención del pensamiento, para ello se le da la pauta de contar del $100 \mathrm{al}$ 1 de tres en tres para atrás. Se le explica que el objetivo es prestar atención de manera voluntaria a una actividad que no está automatizada (contar hacia atrás) para que el pensamiento automático, (por ejemplo: "no voy a ser ca- paz de aguantar sin arrancármelo"), sea sustituido por otro positivo. También se realizó en esta sesión la exposición en imaginación, se practica la situación de estar viendo la televisión, se utilizan todas técnicas trabajadas hasta el momento, siendo la secuencia de este modo: imagina que estás viendo la televisión, sintiendo la tensión para iniciar el movimiento del brazo para arrancarse el pelo, detiene el pensamiento, se da la auto-instrucción positiva, se relaja, observa lo bien que se siente con el pelo, se auto-refuerza. En cuanto a tareas para casa, se le pide a la paciente practicar la técnica de detención de pensamiento, las auto-instrucciones, la exposición en imaginación e in vivo, seguir realizando el auto-registro y reforzarse por todos los logros.

Sexta sesión: En la sexta sesión, comenzamos como en las anteriores y la paciente subraya que no ha habido ningún episodio de arrancamiento. Indica que ha sido capaz de auto-controlarse. Se la refuerza por estos lo- 
gros. Cabe señalar que a esta sesión viene sin pañuelo, y la paciente afirma que poco a poco se va exponiendo a algunas situaciones que previamente evitaba, y que disminuyen los pensamientos negativos y que está mucho mejor su estado de ánimo. Acordamos como tarea para casa seguir con los auto-registros y continuar con la exposición en vivo.

Séptima sesión: En esta sesión se mantiene la remisión de la sintomatología ansioso-depresiva y también hay una remisión de tricotilomanía. Desde la sesión anterior, no se ha producido ningún episodio de arrancamiento. La paciente viene también sin pañuelo, no da muestras de alopecia. Señala que no realiza conductas de evitación. Dados los avances, en esta sesión se lleva a cabo un entrenamiento en prevención de recaídas.

Octava sesión. Se comprueba que se mantiene la remisión de la tricotilomanía. Como elementos importantes a señalar, la paciente refiere que acude con normalidad a la peluquería, se cuida el pelo etc. En cuanto al cuadro ansioso-depresivo, en situaciones de estrés relacionada con la vivienda, juicios pendientes, se mantiene la remisión de la sintomatología. En esta sesión continuamos con el entrenamiento en prevención de recaídas.

Seguimientos: Durante los seguimientos realizados a los tres y seis meses y al año se procedió a la consolidación de los aprendizajes previos.

\section{Resultados}

Desde un punto de vista cuantitativo, las puntuaciones obtenidas a partir del Inventario de situaciones y respuestas de ansiedad, ISRA (Miguel-Tobal y Cano-Vindel, 2002), por la paciente, antes de la intervención, la sitúan dentro del rango de ansiedad severa, con una puntuación centil de 95 en ansiedad total o medida de rasgo general. Respecto a su perfil de respuesta cabe destacar la reactividad en los niveles cognitivo y fisiológico donde obtiene una puntuación centil de 99 , respectivamente, frente al centil 95 alcanzado a nivel motor. Los datos obtenidos en esta prueba referidos a las manifestaciones de la ansiedad, coinciden con los obtenidos en las entrevistas.

Atendiendo a la valoración cuantitativa de los cambios obtenidos tras las sesiones de tratamiento, se comprobó que después del tratamiento existía un descenso importante en todos y cada uno de los niveles de ansiedad valorados por el ISRA, en los tres sistemas de respuesta, cognitivo, fisiológico y motor (Tabla 3).

Puede observarse que fue más importante la disminución de las puntuaciones a nivel motor, donde disminuyó desde un centil 95 (ansiedad severa) hasta por debajo de un centil 5 (mínima ansiedad). En porcentajes, la reduc- ción de las puntuaciones directas que se alcanzó, entre la evaluación pretratamiento y postratamiento, en el nivel general de ansiedad fue del $84.4 \%$ (Tabla 4), alcanzando un valor final de 352 puntos, que correspondería al centil 5 respecto a los baremos de población general.

Tabla 3. Resultados de la evaluación antes y después del tratamiento en puntuaciones directas y centiles

\begin{tabular}{lcccc}
\hline & \multicolumn{2}{c}{ Pre-tratamiento } & \multicolumn{2}{c}{ Post-tratamiento } \\
\cline { 2 - 5 } & PD & PC & PD & PC \\
\hline Cognitivo & 177 & 99 & 45 & 25 \\
Fisiológico & 138 & 99 & 20 & 30 \\
Motor & 102 & 95 & 0 & $\downarrow 5$ \\
Total & 417 & 95 & 65 & 10 \\
\hline
\end{tabular}

Nota. $\mathrm{PC}=$ puntuación centil; $\mathrm{PD}=$ puntuación directa

Tabla 4. Mejorías observadas al finalizar el tratamiento

\begin{tabular}{llc}
\hline & \multicolumn{2}{c}{ Pre/post-tratamiento } \\
\cline { 2 - 3 } & PD & $\%$ \\
\hline Cognitivo & 132 & $74.6 \%$ \\
Fisiológico & 118 & $85.5 \%$ \\
Motor & 102 & $100 \%$ \\
Total & 352 & $84.4 \%$ \\
\hline
\end{tabular}

Nota. $\mathrm{PD}=$ puntuación directa

Con respecto a la conducta de arrancarse el pelo, los resultados a lo largo de estas 8 sesiones de intervención, ponen de manifiesto una importante mejoría: se paso de 163 pelos arrancados, durante un intervalo de una a dos horas, a no tener ningún episodio de arrancamiento después del tratamiento y en los seguimientos. Las conductas de evitación desaparecieron (tras la terapia, iba sin pañuelo, acudía a la peluquería, iba sentada en el autobús, se paraba a hablar en la escalera con alguien situado por encima de su cabeza, se agachaba para jugar con el perro de otra persona, respondía a preguntas sobre su pelo...). Además, se podrían considerar otros resultados positivos como la remisión de sintomatología ansioso-depresiva, y el restablecimiento de actividades rutinarias, distractoras, sociales y familiares. La paciente empezó a afrontar los juicios relacionado con la vivienda, tolerando el malestar que estas situaciones le producían.

Tras las pertinentes sesiones de seguimiento a los $3 \mathrm{y}$ 6 meses, la paciente se mantiene asintomática, refiriendo un alto nivel de satisfacción con los resultados obteni- 
dos. A su vez, indica que su autoestima y seguridad en sí misma también han ido mejorando al sustituir un estilo de afrontamiento de los problemas diarios basado en la preocupación por otro basado en la identificación y la solución de los mismos.

\section{Discusión}

Como se ha señalado, este trabajo se centra en presentar las estrategias de intervención cognitivo-conductuales utilizadas en un caso de tricotilomanía que estaba dando lugar a consecuencias muy negativas en la vida de la paciente. Se comprueba que dichas estrategias resultaron eficaces y que con un número breve de sesiones ( 8 sesiones de tratamiento y 3 sesiones de seguimiento) se consiguieron mejorías muy importantes disminución drástica de los episodios y la frecuencia del arrancamiento del pelo, disminución importante de la ansiedad y normalización del estado de ánimo. Estos resultados apoyan la eficacia del procedimiento seguido y están en la línea de estudios previos sobre el tema (Ninan et al., 2000). Por tanto, se pone de manifiesto la conveniencia de utilizar técnicas de tratamiento cognitivo-comportamentales que cuenten con apoyo empírico para el manejo de este problema y su viabilidad en un contexto de sanidad pública.

Cabe destacar también el proceso seguido en la aplicación de las técnicas utilizadas en la intervención a través de las diferentes sesiones y como en cualquier proceso de terapia, hay que señalar que la colaboración de la paciente, realizando las tareas para casa y asistiendo a las sesiones con total compromiso y regularidad, tuvo un papel importante en la efectividad de la intervención.

Este trabajo tiene importantes limitaciones, la fundamental que se trata de un estudio de caso, con todas las amenazas a la validez externa que tienen este tipo de estudios. También hubiera sido deseable llevar a cabo una evaluación contando con un mayor número de instrumentos y contar con un evaluador independiente ajeno por completo a la aplicación del tratamiento. Sin embargo, deseamos subrayar que en el contexto de sanidad pública en el que se llevó a cabo el tratamiento, no es posible muchas veces contar con tiempo y recursos adicionales debido a la notable presión asistencial. Además, un objetivo fundamental del trabajo era precisamente, mostrar que, aún con dificultades, sí es posible aplicar este tipo de procedimientos de evaluación y de tratamiento con respaldo empírico.

Sin lugar a dudas siguen quedando pendientes muchos problemas no resueltos en este trastorno a los que se debería prestar atención. Esto se debe en gran parte a que sólo muy recientemente se ha reconocido la impor- tante discapacidad asociada a la tricotilomanía, a la negación y ocultamiento que hacen de la misma algunos pacientes y a que este trastorno se produce con mayor frecuencia de la que se pensaba (Duke et al., 2010). En cualquier caso, es necesario progresar en este ámbito llevando a cabo ensayos clínicos controlados, analizando también el papel que pueden desempeñar en el mantenimiento del problema los estados de ánimo negativos asociados o las situaciones estresantes, como ocurría en nuestra paciente. Una mayor comprensión de este trastorno resulta esencial para poder diseñar mejores protocolos de tratamiento que puedan ser sometidos a prueba y que resulten de utilidad para todas aquellas personas que sufran este problema. Un ejemplo paradigmático de los avances que se pueden producir en este campo es el trabajo de Roberts, O'Connor y Bélanger (2013) acerca de la importancia que puede tener la regulación emocional en estos problemas de conductas repetitivas centradas en el cuerpo.

\section{Referencias}

Azrin, N. H., \& Nunn, R. G. (1973). Habit reversal: a method of eliminating nervous habits and tics. Behavioral Research and Therapy, 11, 619-628.

Cardenal, V., Sánchez, M. P., y Ortiz-Tallo, M. (2007). Adaptación y baremación al español del Inventario Clínico Multiaxial de Millon-III, MCMI-III. Madrid: TEA Ediciones.

Christenson, G. A., Ristvedt, S. L., y Mackenzie, T. B. (1993). Identification of trichotillomania cue profiles. Behaviour Research and Therapy, 31, 315-320.

Cohen, L. J., Tein, D.J., y Simeon, D. (1995). Clinical profile, comorbidity and treatment history in 123 hair pullers: a survey study. J Clin Psychiatry, 56, 319-326.

Diefenbach, G. J., Tolin, D. F., Hannan, S., Crocetto, J., y Worhunsky, P. (2005). Trichotillomania: impact on psychosocial functioning and quality of life. Behaviour Research and Therapy, 43, 869-884.

Duke, D. C., Keeley, M. L., Geffken, G. R., \& Storch, E. A. (2010). Trichotillomania: a current review. Clinical Psychology Review, 30, 181-193.

Elliott, A. J., \& Fuqua, R. W. (2000). Trichotillomania: Conceptualization, measurement, and treatment. Behavior Therapy, 31, 529-546.

Enos, S., \& Plante, T. (2001). Trichotillomania: An overview and guide to understanding. Journal of Psychosocial Nursing and Mental Health Service, 39, 10-16.

Hallopeau, M. (1989). Alopecia par grattage (trichomanie ou trichotillomanie). Ann Dermatol Syphil, 10, 440-441.

Kraemer, P. A. (1999). The application of habit reversal in treating trichotillomania. Psychotherapy, 36(3), 298-304.

Kress, V., Kelly, B., \& McCormick, L. (2004). Trichotillomania: assessment, diagnosis and treatment. Journal of counseling and development, 82, 185-190.

Lerner, J., Franklin, M., Meadows, E. A., Hembree, E., \& Foa, E. B. (1998). Effectiveness of a cognitive behavioural treatment 
program for Trichotillomania: An uncontrolled evaluation. Behavior Therapy, 29, 381-395.

Lochner, C., Seedat, S., Toit, P., Nel, D., Niehaus, D., Sandler, R., \& Stein, D. (2005). Obssesive compulsive disorder and Trichotillomania: a phenomenological comparison. BMC Psychiatry, 5(1), 2.

Miguel-Tobal, J. J., y Cano-Vindel, A. R. (2002). Inventario de Situaciones y Repuestas de Ansiedad, ISRA. TEA ediciones.

Millon, Th. (1994). Millon Clinical Multiaxial Inventory-III, MCIM-III. Minneapolis: Nacional Computer Systems. Adaptación española: Cardenal, V., Sanchez, M. P. y Ortiz-Tallo, M. (2007). TEA ediciones.

Papadopoulos, A., Janniger, C., \& Schwartz, R. (2003). Trichotillomania, a review. Int J Dermatol, 42(5), 330-334.
Pérez-Elizondo, A. D., Pineda-Pineda, J. A., \& Arellano-Flores, J. (2012). Trichotillomania: searching an unnoticed pathology. Revista Argentina de Dermatología, 93, 65-76.

Roberts, S, O'Connor, K., \& Bélanger, C. (2013). Emotion regulation and other psychological models for body-focused repetitive behaviors. Clinical Psychology Review, 33, 745-762

Shusterman, A., Feld, L., Baer, L., \& Keuthen, N. (2009). Affective regulation in trichotillomania: evidence from a large-scale internet survey. Behaviour Research and Therapy, 47, 637-644.

Woods, D. W., Flessner, C., Franklin, M. E., Wetterneck, C. T., Walther, M. R., Anderson, E. R., \& Cardona, D. (2006). To understand and to treat the trichotillomania: what we Know and what we do not Know. Psychiatric Clinics of North America, 29, 487-501. 